\title{
A Hybrid Approach for Blur Detection Using Naïve Bayes Nearest Neighbor Classifier
}

\author{
Harjot Kaur \\ Dept. of Computer Science and Engineering, Sri Guru Granth Sahib World University, Punjab, India \\ E-mail: harjotkaur94639@g mail.com \\ Mandeep Kaur \\ Dept. of Computer Science and Engineering, Sri Guru Granth Sahib World University, Punjab, India \\ E-mail: mandeepkaur.dhot@gmail.com
}

\begin{abstract}
Blur detection of the partially blurred image is challenging because in this case blur varies spatially. In this paper, we propose a blurred-image detection frame work for auto maticallQy detecting blurred and nonblurred regions of the image. We propose a new feature vector that consists of the information of an image patch as well as blur kernel. That is why it is called kernelspecific feature vector. The information extracted about an image patch is based on blurred pixel behavior on local power spectrum slope, gradient histogram span, and maximum saturation methods. To make the features vector useful for real applications, kernels consisting of motion-blur kernels, defocus-blur kernels, and their combinations are used. Gaussian filters are used for filtering process of extracted features and kernels. Construction of kernel-specific feature vector is followed by the proposed Naïve Bayes Classifier based on Nearest Neighbor classification method (NBNN). The proposed algorithm outperforms the up-to-date blur detection method. Because blur detection is an initial step for the de-blurring process of partially blurred images, our results also demonstrate the effectiveness of the proposed method in deblurring process.
\end{abstract}

Index Terms - Blur detection, feature extraction, motion blur, defocus blur, support vector machine (SVM), NBNN, deblurring.

\section{INTRODUCTION}

In recent years, the commercialization of mobile cameras increases the number of casual photographers that means people are allowed to capture a huge quantity of photos without difficulty. The increase in a number of casual photographers increases the number of failure photographs containing noisy, blurred, and unnaturallycolored images. That is why an automatic system is required to avoid and correct the photographs with low quality [1].

Digital cameras are integrated with auto-exposure, automatic white balance, and noise reduction capabilities to resolve exposure, color, and noise issues; but handle image blur only in an imperfect manner. For example, depth of specific scene can be focused with an autofocus function, but it can't capture things at different depths sharply at the same time. Due to the incompatibilities of an autofocusing feature of cameras', in personal photo collections, defocused blur images are commonly seen.

We can discard bad image data at the source itself by integrating image blur detection in a camera. Blur detection approach can also help in restoration (deblurring) process. One of the challenges of image deblurring is to recover information from available blurred data through efficient and re liable algorithms. Deblurring of fully blurred image is easier than partially blurred images where only a part or few objects of an image are blurred. Deblurring of whole partially blurred image is costly and also produces wrong results. Therefore, there is a need to detect the blurred region of the partially blurred image to restore it where restoration process only applies on the blurred region (not on unblurred region) of the image [14].

Other applications on which blur detection can be applied are- object extraction, scene classification, image quality assessment, forensic investigation (detects criminals from low-quality camera's footage that has some blurred content) etc. In fact, automatic blur detection can replace most of the human operator work of extracting useful information from the blurred image and vast applications of blur detection increase the need of research in this topic.

However many techniques have been proposed for the blur detection such as Edge and sharpness analysis [3], Gaussian blur kernel detection [1], Discrete Wavelet Transform (DWT) [13], Maximu m Likelihood (ML) Low depth of field (DOF) image auto-segmentation [23], kernel based SVM classifier etc. but there arises some problems such as: (1) Techniques are easy to apply on simpler images but less effective for complex images, (2) These techniques are effective for small databases but it is difficult to sample the large database, (3) The techniques are only effective to specific type of blur (either motion blu r or out-of-focus blur images), (4) User interaction is needed for correct estimations. So there is a need to propose the suitable technique which can resolve all these problems.

In this paper, we proposed a new technique that resolves above-mentioned problems, and results in 
improved blur detection accuracy. The contribution of this paper is threefold. First, we propose new feature vector based on three features and combination of motion and defocus blurred kernels. Second, we use a hybrid approach (NBNN) to classifying the input image regions into blurred and unblurred regions. Third, we apply deblurring technique on our results to deblur pixels only inside the blur region.

We provide results for defocus blur and motion blur images (that are the basic types of blur). Most of the existing blur related researches are based on only motion and defocus blur.

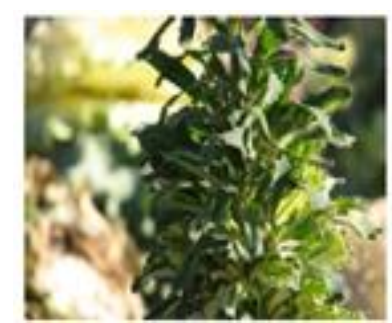

(a)

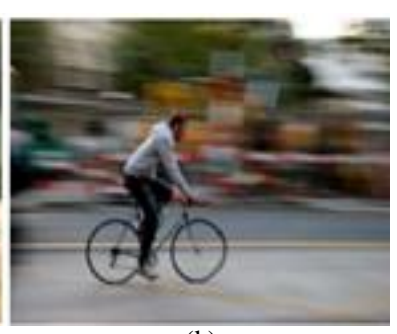

(b)
Fig.1. Types of blur: (a) Defocus Blur (b) Motion Blur

The Structure of paper: This Introduction section covers the basic introduction to our topic, applications of blur detection, existing problems and basic structure of our proposed method. Organization of rest of the paper is as follow: (a) Section II covers related literature review or work, (b) Section III covers proposed method that describes the blur features, blur kernels, classifier, and structure used to execute our proposed work, (c) Section IV covers the experimental results of proposed method of blur detection. It also presents the comparison in terms of accuracy of proposed method with popular existing methods. (d) Results after deblurring the blurred image using blur detection are presented in Section V (e) Conclusion and Future work are presented in Section VI and Section VII respectively.

\section{RELATED WORK}

During the last few decades, topics related to the blurred image have been studied deeply in the field of computer vision and image processing. In this section, we review general blur detection methods.

The shape of an object is due to its edges. An image is said to be sharp if objects and their shapes can be perceived correctly. For example object's face in an image looks clear only when we can identify eyes, ears, nose, lips, forehead etc. very clearly. But factors like blurring (where an image is blurred through photoediting tools or filters), environment condition, relative motion between camera and scene, low-quality camera etc. reduce the edge content and makes the transition between colors very smooth. Blur can be detected directly through edge and sharpness analysis. A sharp object contains only step edges, but step edges turn into ramp edges when that object gets blurred. Therefore, measurement of the sharpness or blurriness of edges can be used to detect blur. Chung et. al [2] used gradient magnitude and edge direction to measure degree of blurriness in the image. Firstly, they fitted gradient magnitude and edge direction into a normal distribution. Then, they computed the standard deviation of normal distribution along with gradient magnitude to measure edge width and edge magnitude that make blur measurement more reliable. In contrast, measurement of the degree of blur through the thickness of object contours is performed in the paper by Elder and Zucker [3]. They modeled focal blur model by a Gaussian blur kernel and then used the first and second order derivative of Gaussian filters (steerable) [4] to calculate the response that describes the degree of blur in an image. This method is used for only local edge estimation over a wide range of contrast and local blur scale and requires the only second moment of the sensor noise as input parameters. While for multi-scale blur estimation Zhang and Bergholm [5] defined Gaussian Difference Signature that functions similarly to the first-order derivative of Gaussian.

Bayes discriminant function can be constructed based on the statistic of the gradients that perform mean and standard deviation on both blurred and sharp regions [6]. In the distribution blurred region of an image has a smaller value of mean and standard deviation than a sharp region of the image. This concept helps to detect the blurred region in an images for further de-blurring processes. Naive Bayesian classifier can also integrate blur feature set obtained from different do mains based on the posterior score. For example, Shi Jianping et al. [7] proposed a new blur feature set (in multiple domains) that is based on Gradient Distribution as well as Frequency Domain and Local filters. Similarly, Renting Liu et al. [8] proposed feature set based on image color, gradient and spectrum information. These features are used by Bayes Classifier to detect spatially-varying blur and type of blur.

Blur detection method based on the no-reference (NR) block do not require the original sharp image to measure the degree of blur. Therefore, this method has less complexity and high robustness compared with edge based blur metrics. Blur metrics can be computed by averaging macro blocks of local blur; and content dependent weighting scheme reduces the texture influence [9]. Based on human blur perception for contrast-varying values, Niranjan D. Narvekar et al. [10] presented a no-reference image blur metric that utilizes a probabilistic model which is used to evaluate the probability of blur detection at each edge in the image).

Lowest directional high frequency energy is used for motion blur detection and has less computational cost because estimation of point spread function is not required [11]. Along the motion blur direction, high frequency energy decrease where energy refers to the sum of the squared derivative of image [12].

Discrimination between blurred and non-blurred image regions (based on the gradient distribution) can be conducted using SVM classifier [13]. Proposed method performs wavelet decomposition on the input image to 
extract features in wavelet space and constructs gradient histograms on which probabilistic SVM is applied to detect blurred region. In [14], kernel-specific feature vector consisting of the multiplication of the variance of the filtered gradient of image patch and the filtered kernel is classified by SVM. This paper is related to most of our proposed work. Their results showed higher accuracy for defocus blur compared with motion blur. We present the comparison of results between our method and their method in the results section.

Low Depth of Field (DoF) is a photography technique which provides clear focus only on a specific object and is used to detect and remove blur at the source. Autosegmentation of low DoF images can be conducted through- pixel-wise spatial distribution of the high frequency components [15], morphological filters [16], localized blind deconvolution that produces a focus map [17], multi-scale context-dependent approach [18], the ratio of wavelet coefficients [19] and so on.

After the detection of the blurred region, we can apply the deblurring techniques on it without affecting the unblurred region. Inverse Filter [1, 21], Least-Square Filter [21] and Iterative Filters [22] are used to restore image when prior information about the degrading system is available. While, a prior blur identification [21], ARMA parameter estimation, Non-parametric deterministic image-based restoration [23] and Maximu m-Likelihood (ML) [24] methods are used, when prior information about the degrading system is not available. These methods work well on spatiallyinvariant blur. To tackle the partial blur problem, transparency based MAP model can be used [26]. But this method requires user interaction for better results whereas our proposed method does not require user interaction.

\section{PROPOSED METHOD}

Due to the diversity of the natural images, we propose a frame work to detect blur in the partially blurred images. The basic flow chart of our system is given in Fig. 2 and is explained in brief in the following:

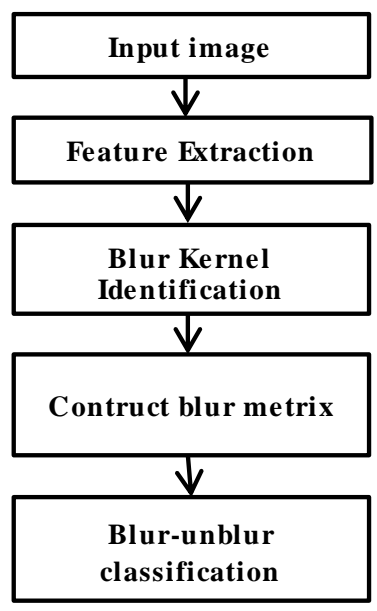

Fig.2. Basic Flow Chart for blur detection a) For each input image, first, extract features of the image.

b) Based on the information of extracted features select blur kernel from the pool of kernels consisting of motion blur kernels, defocus blur kernels and their combinations.

c) Construct the kernel-specific feature vector or blur matrix from step (a) and (b).

d) On the basis of the blur matrix detection of blurred pixels is performed i.e. hybrid NBNN classifier is used to classify the blurred and non-blurred regions of the image.

\section{A. Feature Extraction}

There are three different features developed and combined in our system. These features are derived by analyzing the visual and spectral clues from images. These features are described in following:

\section{Local Power Spectrum Slope}

The strength of change in an image is defined by its frequency components. Sharp edges of the image have high frequency value. Power spectrum uses frequency components to detect the blurred and unblurred regions. Some high frequency components of the blurred image are absent due to the low-pass-filtering characteristic of the blurred regions. So, for blurred region amplitude spectrum slope tends to be steeper than unblurred region.

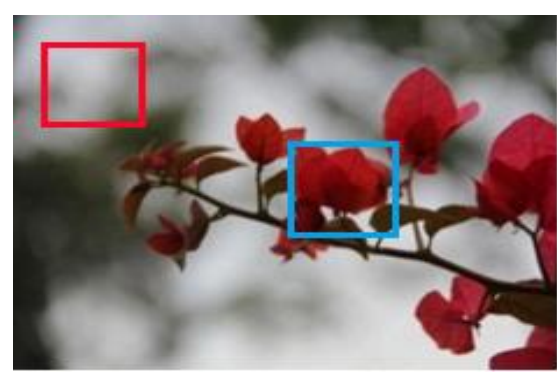

(a)



(b)

Fig.3. (a) Input image (b) Power Spectrum Slope of a blurred patch (shown in a rectangle of red color) and unblurred region (shown in a rectangle of blur color) are shown where blurred and unblurred region have different values.

It is not reliable to simply set a threshold value for the blur estimation because an image may have multiple objects with the different type of edges or boundaries. On the basis of this observation, we first compute the global measure of the slope of the power spectrum $\left(\alpha_{0}\right)$ for the 
whole image. Then we compare power spectrum $\left(\alpha_{\mathrm{p}}\right)$ computed in each local block p with $\alpha_{0}$. If $\alpha_{\mathrm{p}}$ is much larger than $\alpha_{0}$, it is quite possible that this block is blurred.

\section{Gradient Histogram Span}

Gradient magnitude distribution helps as important visual evidence in blur detection process. Blurred regions have a small gradient magnitude (or in log gradient distribution have shorter tails) while unblurred regions have large gradient magnitude. As shown in Fig. 4, in a case of a blurred region, the number of pixels having zero gradient value is large. That is why it has a short tail and high peak at zero gradient value while the unblurred region has a heavy tail and less mass at zero gradient value.

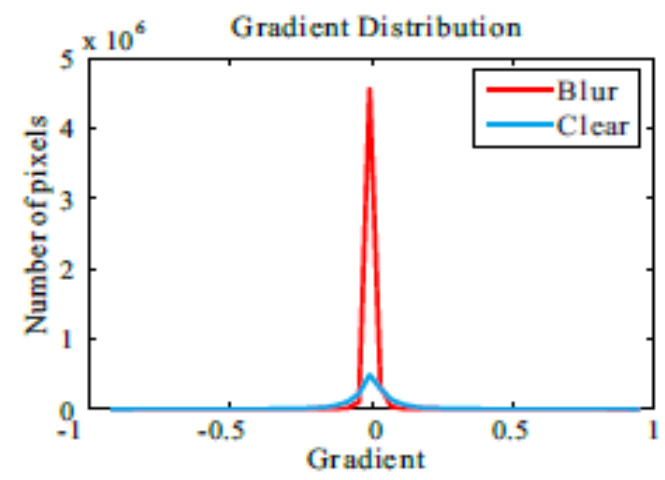

Fig.4. Gradient Histogram Span of the blurred patch (represented in red color) and unblurred region (represented in blur color) is shown where the blurred region has strong peak at zero gradient value i.e has small tail while the unblurred region has heavy tail.

\section{Maximum Saturation}

The maximum value of saturation of blurred regions is correspondingly expected to be smaller than that of unblurred regions. Based on this observation, we first compute the maximum saturation value $\left(\max \left(\mathrm{S}_{0}\right)\right)$ of the whole image. Then within each patch $p$, we compute saturation $\mathrm{Sp}$ for each pixel and find the maximum saturation value $(\max (\mathrm{Sp}))$ that is compared with $\max \left(\mathrm{S}_{0}\right)$. If $\max (\mathrm{Sp})$ is less than $\max \left(\mathrm{S}_{0}\right)$ than the patch is blurred otherwise not.

\section{B. Blur Kernel Identification}

To identify the blur for an input image, we perform blur edge analysis and derive the following attributes of the blur kernel:

- Blur kernel of defocus blur type are isotropic in nature i.e. edges in every direction are s moothened.

- Blur kernel of motion blur type are an is otropic i.e. edges with the same direction as the motion direction will be the least affected while edges perpendicular to the motion direction will be blurred most severely.

- Blur kernel shows no effect when applying to a flat region without illuminance changes. In fact, flat regions contain no useful information for blur detection.

\section{Construct Blur Matrix}

The kernel-specific feature vector (or blur matrix) is composed of the multiplication of the variance of filtered kernel (obtained using subsection B methods) and the variance of filtered patch features (obtained using subsection $\mathrm{C}$ methods).

\section{Blur-unblur Classification}

Through hybrid approach complex problems can be solved by stepwise decomposition. Specific hierarchical levels (on the basis of concept granularity) are defined in intelligent hybrid systems. Therefore to discriminate unblurred and blurred regions, based on proposed feature vector, we use NBNN classifier where Naïve Bayes classifier is used for training purpose and nearest neighbor approach is used to improve the accuracy through additional testing on the neighborhood pixels.

When only Naïve Bayes classification approach is used to discriminate the blurred and unblurred region, it detects defocus blur more accurately than motion blur [7]. Therefore, we use nearest neighbor classification approach, on neighborhood pixels to increase the accuracy of the motion blur detection method because in the motion blurred image blurring color of one pixel spread to its neighborhood that will increase its color similarity to its neighboring pixels.

\section{EXPERIMENT AL RESULTS}

The performance of the proposed method is estimated on the public dataset of 1000 image accessible at [29] with its ground truth images. Out of 1000 blurred images, 296 images are motion blurred images while others are defocused blurred images. The proposed method is compared with the method developed by Y. Pang et al. [14] named as SVM classifier. In their method, kernel specific feature vector based on gradient magnitude and blur kernel; and SVM classifier is adopted. It is the most successful existing method because their experimental results outperform the method proposed by Chakrabarti et al. [20], Liu et al. [8], and Su et al. [27]. It is enough to compare our proposed method with SVM classifier results. To show the need for the hybrid approach we also compare our results with results of Naïve Bayes classifier results obtained by Shi et al. [7] openly available at http://www.cse.cuhk.edu.hk/leojia/projects/dblurdetect/.

Results (in the form of images) of SVM classifier, Naïve Bayes classifier and proposed method on motion blur and defocus blur images along with their ground truth image are shown in Fig. 5 and 6. The output image is displayed in black and white color where blurred pixels are represented with white color while unblurred pixels with a black color to make the differentiation between the blurred and unblurred part of an image clearer.

To compute the accuracy of these methods ground truth image are compared with the obtained results to measure how many pixels are classified accurately. The accuracy of SVM classifier, Naïve Bayes classifier, and proposed method is shown in table land reasons for their 
different behavior are given below.

Naïve Bayes classifier [7] has wrongly detected most of the unblurred pixels as blurred because this classification method does not consider the neighborhood pixels during classification process and its feature set was not directly based on the kernel as used in SVM and our proposed method. However, it detected blurred pixel more accurately than SVM classifier because its feature set is based on multiple blur features and is tested at multiple scales to avoid ambiguous results.

SVM classifier used in paper [14] used feature vector that is based on gradient distribution and blur kernels. Accuracy of this method is more than Naïve Bayes classifier because it uses kernel specific feature set formed by multiplying the variance of filtered gradient distribution and the filtered blur kernel where the measure of variance reduce the chance of error (less amount of unblurred pixels are detected as blurred) during the classification process. Results of proposed method (Naïve Bayes Nearest Neighbor Classifier) are more accurate because its classification results are based on multiple blur features and a combination of blur kernels (defocus and motion blur kernels), and neighborhood pixels are also considered to improve the classification process.

As shown in table 1, the accuracy of SVM and Naïve Bayes classifier is different for motion and defocus blur. In fact, the accuracy of defocus blur images is approximately $10 \%$ more than motion blur images. But the accuracy of motion blur images and defocus blur images is approximately same in the case of proposed method results.

Table 1. Comparison in Accuracy

\begin{tabular}{|c|c|c|c|}
\hline \multirow{2}{*}{ Method } & \multicolumn{3}{|c|}{ Accuracy } \\
\cline { 2 - 4 } & Motion blur & Defocus blur & Average \\
\hline $\begin{array}{c}\text { Proposed } \\
\text { method }\end{array}$ & 64.12 & 65.2 & 64.7 \\
\hline $\begin{array}{c}\text { SVM classifier } \\
{[14]}\end{array}$ & 49.8 & 57.6 & 54.4 \\
\hline $\begin{array}{c}\text { Naïve Bayes } \\
\text { Classifier [7] }\end{array}$ & 41.2 & 53.3 & 51.1 \\
\hline
\end{tabular}

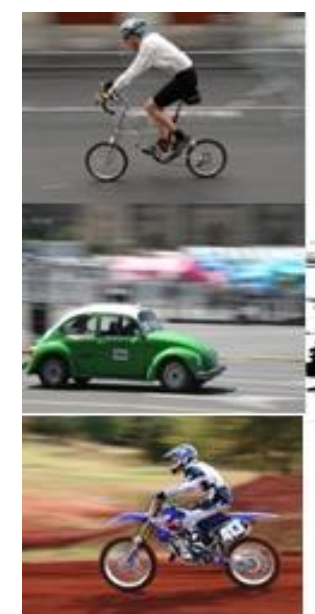

(a) Input Image

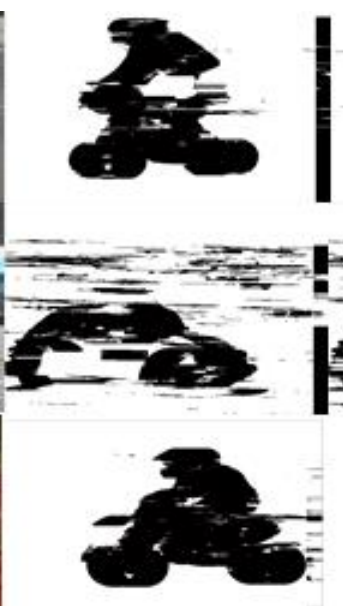

(b) Proposed Method
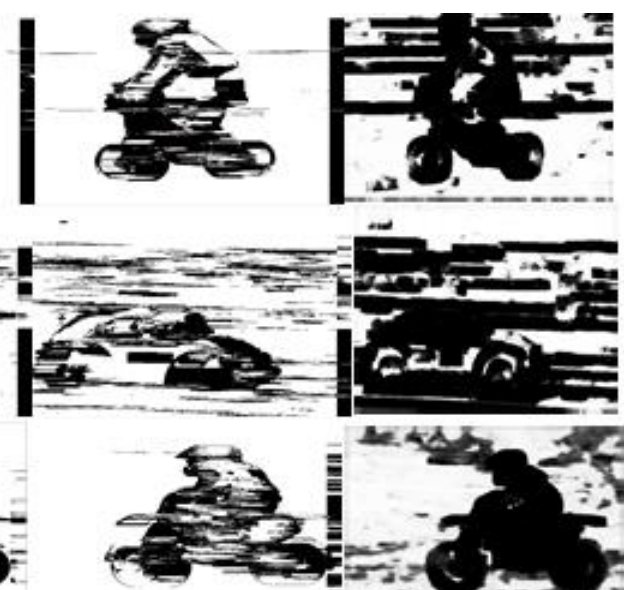

(c) SVM Classifier

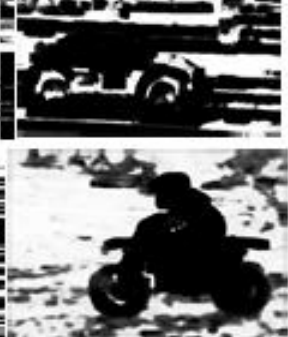

(d) Naïve Bayes
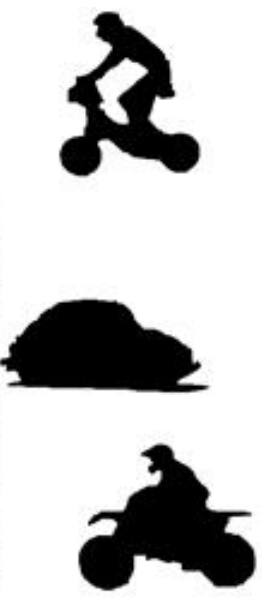

(e) Ground Truth

Fig.5. Results of Motion Blur Detection

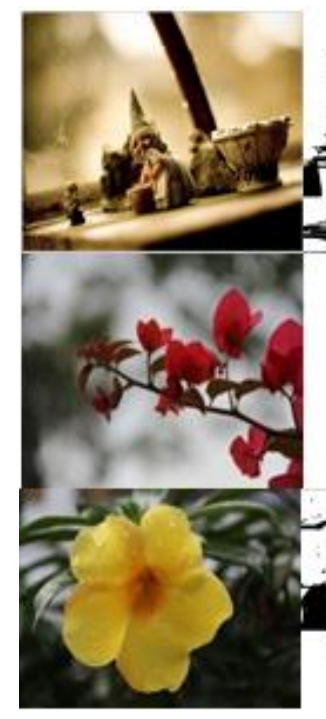

(a) Input Image

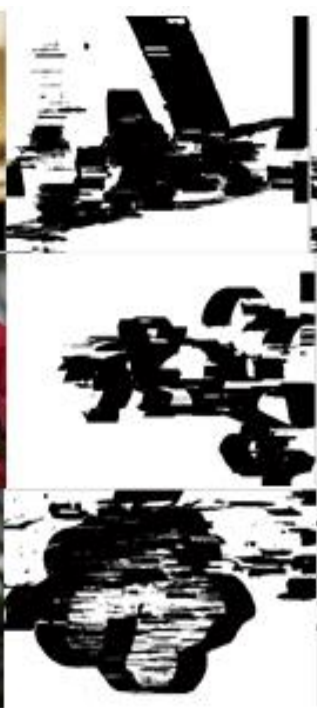

(b) Proposed Method

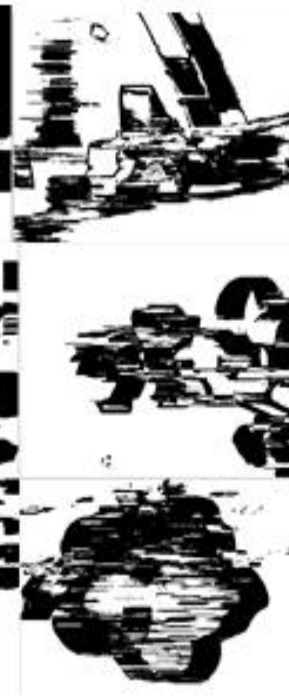

(c) SVM Classifier

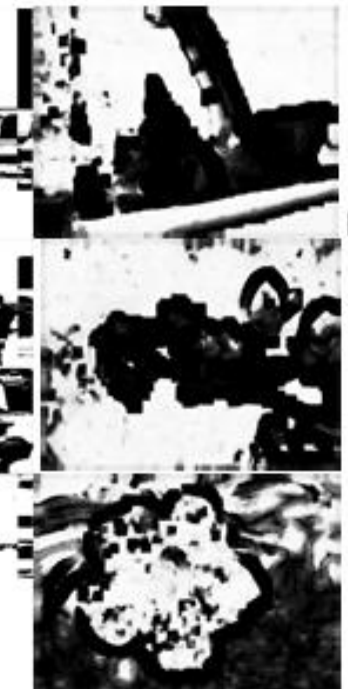

(d) Naïve Bayes



(e) Ground Truth

Fig.6. Results of Defocus Blur Detection 


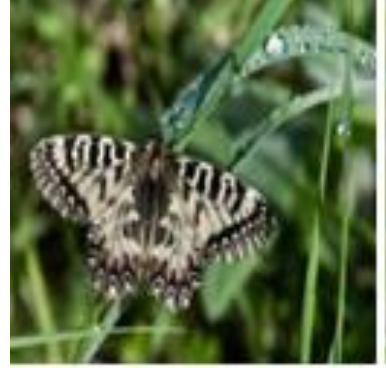

(a)

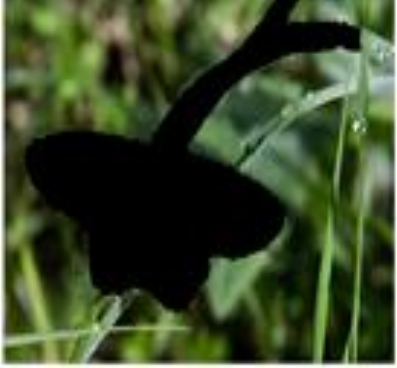

(b)

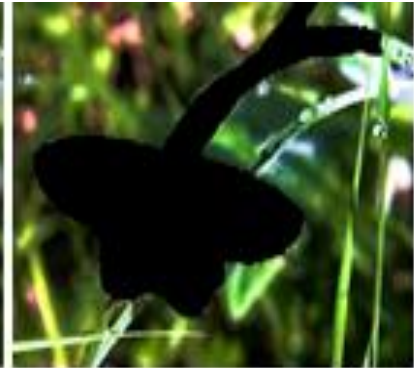

(c)

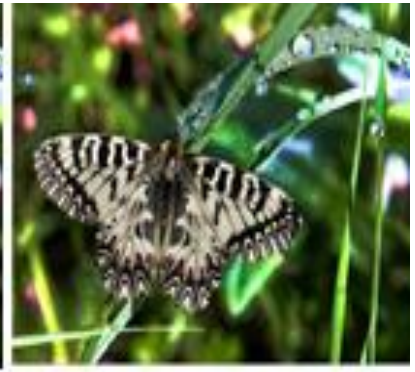

(d)

Fig.7. Restoration results of defocus blur image: (a) Input Image (b) Blur segmented image where the black region is the segmented unblurred region (c) Deblurring results of the blurred region (d) Final restored image.

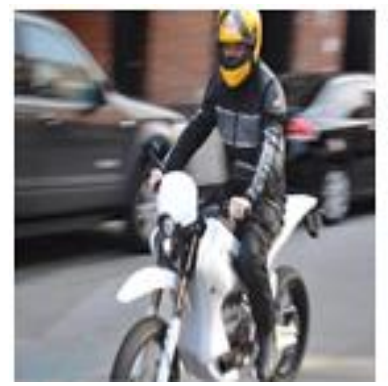

(a)

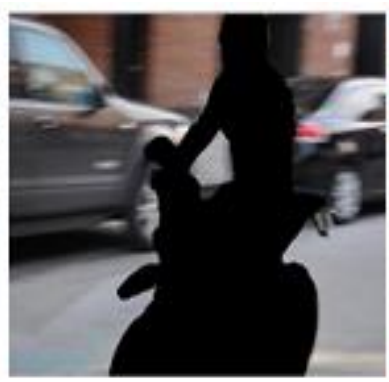

(b)

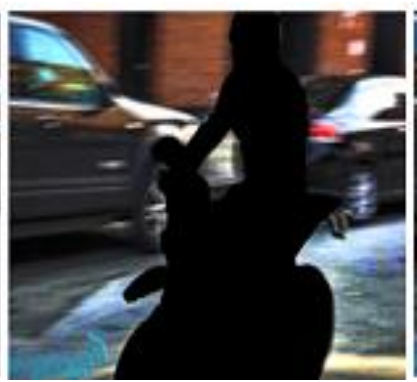

(c)

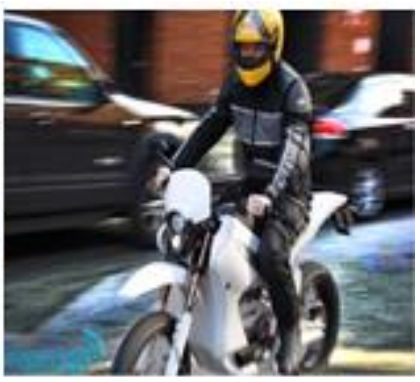

(d)

Fig. 8. Restoration results of motion blur image: (a) Input Image (b) Blur segmented image where the black region is the segmented unblurred region (c) Deblurring results of the blurred region (d) Final restored image.

\section{IMAGE DEBLURRING RESULTS}

Several computer vision applications can be benefitted from our blur detection task. We show blur segmentation and deblurring in this section. Existing non-blind deconvolution mixes foreground and background under different motion but our objective is to deblur pixels only inside blur masks. To perform deblurring we first perform blur segmentation followed by deblurring process explained in the following:

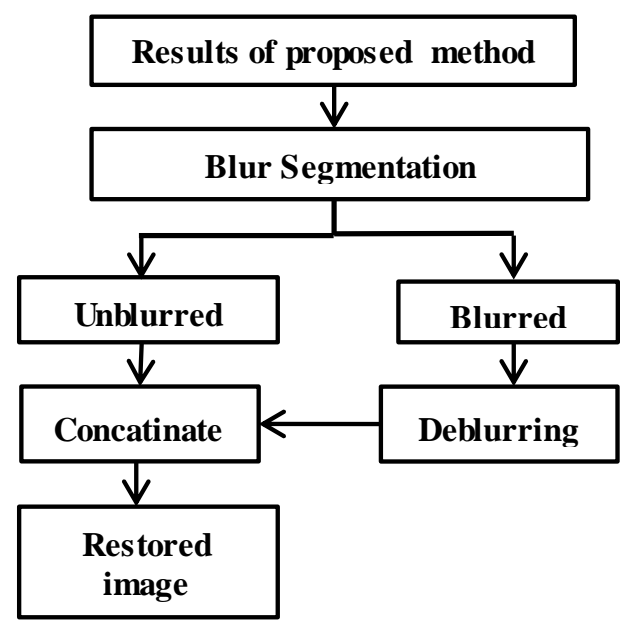

Fig.9. Basic Deblurring Flow Chart

Step 1- Blur Segmentation: With our proposed method, it is possible to segment images into the blur and clear regions. In Fig. 7 and Fig. 8, (a) part shows the defocus and motion blurred input image respectively and (b) part shows the results of blur segmentation process that subtract the detected unblurred reg ion from the input image. That is why; the unblurred region is displayed in black color.

Step 2- Deblurring of blurred region: With the help of blur segmentation step, we subtract the unblurred region of the blurred image and apply image deblurring method on the blurred part of an image (results of restored blurred region are presented in Fig. 7(c) and 8(c)). Finally, we concatenate the original unblurred region and the recovered blurred region of the blurred image (that is presented in Fig. 7(d) and 8(d)).

\section{CONCLUSION}

In this paper, we have developed a new kernel specific feature based on more than one blur detection features. The combination of motion blur kernels and defocus blur kernels also plays an important role in the construction process of proposed blur detection feature vector. Instead of using a single classifier, we use NBNN (hybrid) classifier.

We construct extensive experiments with training and testing dataset of 1000 images and use them to test the accuracy of our classification algorith $\mathrm{m}$. To estimate the effectiveness of proposed method, we compare it with existing method (SVM and Naïve Bayes classifier). Most of the existing methods provide good blur detection results only for specific type of blur. But our method works well on both types of blur.

To show how blur detection can be used for deblurring process, after the detection of the blurred region of an 
image we segment the blurred part of the image from the unblurred part and existing deblurring method is applied only on segmented blurred part. Then the original unblurred part of the image is concatenated with the results of deblurring method.

\section{FUTURE WORK}

Our classification results inevitably contain errors in natural images due to the similarity of the blurred and low-contrast regions. So, the main problem of future work is to make our system work more robust. Other future works involve segmentation of images into layers with different blur extent for further utilization of the blur features. To make our classification results more effective and robust, application of different classification methodologies is also possible.

\section{REFERENCES}

[1] Bovik and J. Gibson, Handbook of image and video processing, Academic Press, Inc. Orlando, FL, USA, 2000.

[2] Y. Chung, J. Wang, R. Bailey, S. Chen and S. Chang, "A nonparametric blur measure based on edge analysis for image processing applications", IEEE Conference on Cybernetics and Intelligent Systems, vol. 1, 356 - 360, 2004.

[3] J. H. Elder and S. W. Zucker, "Local scale control for edge detection and blur estimation," IEEE Conf. on Pattern Analy sis and Machine Intelligence, vol. 20, no. 7, pp. 699-716, 1998.

[4] W. T. Freeman and E. H. Adelson, "The design and use of steerable filters," IEEE Conf. on Pattern Analysis and Machine Intelligence (PAMI), vol. 13, no. 9, pp. 891906, 1991.

[5] W. Zhang and F. Bergholm, "Multi-scale blur estimation and edge type classification for scene analysis," International Journal of Computer Vision, vol. 24, no. 3, pp. 219-250, 1997.

[6] Jaeseung Ko and Chan gick Kim, "Low cost blur image detection and estimation for mobile devices," IEEE International Conference on Advanced Computing Technologies (ICACT), vol. 3, pp. 1605-1610, 2009.

[7] J. Shi, L. Xu. and J. Jia, "Discriminative blur detection features," IEEE Int. Conf. Comput. Vis. Pattern Recognit., pp. 2965-2972, 2014.

[8] R. Liu, Z. Li and J. Jia, "Image partial blur detection and classification," CVPR, pp. 1-8, 2008.

[9] Liu Debing, Chen Zhibo, Ma Huadong, Xu Feng and Gu Xiaodong, "No reference block based blur detection," Quality of Multimedia Experience International Workshop, pp. 75-80, 2009.

[10] Niranjan D. Narvekar and Lina J. Karam, "A nonreference image blur metric based on the cumulative probability of blur detection (CPBD)," IEEE Trans. on Image Processing, pp. 2678-2683, 2011.

[11] Xiaogang Chen, Jie Yang, Qiang Wu and Jiajia Zhao, "Motion blur detection based on lowest directional highfrequency energy," IEEE International Conference on Image Processing (ICIP), pp. 2533-2536, 2010.

[12] X. Zhu, S. Cohen, S. Schiller and P. Milanfar X. Zhu, "Estimating spatially varying defocus blur from single image," IEEE Trans. on Image Process., pp. 4879-4891, 2013.
[13] V. Kanchev, K. Tonchev and O. Boumbarov, "Blurred image regions detection using wavelet-based histograms and SVM," IEEE International Conference on Intelligent Data Acquisition and Advanced Computing Systems (IDAACS), pp. 457-461, 2011.

[14] Y. Pang, H. Zhu, Xinyu Li and Xuelong li, "Classifying discriminative features for blur detection," IEEE Trans. on Cybernetics, pp. 2168-2267, 2015.

[15] C. S. Won, K. Pyun and R. M. Gray, "Automatic object segmentation in images with low depth of field," ICIP, 3, pp. 805-808, 2002.

[16] C. Kim, "Segmenting a Low-Depth-of-Field image using morphological filters and region mergin," IEEE Transactions on Image Processing, no. 14, pp. 1503-1511, 2005.

[17] L. Kovacs and T. Sziranyi, "Focus area extraction by blind deconvolution for defining regions of interest," PAMI, vol. 29, no. 6, pp. 1080-1085, 2007.

[18] J. Z. Wang, J. Li, R. M. Gray and G. Wiederhold, "Unsupervised multiresolution segmentation for images with low depth of field," PAMI, 23,pp. 85, 2001.

[19] R. Datta, D. Joshi, J. Li and J. Z. Wang, "Studying aesthetics in photographic images using a computational approach,"ECCV ,pp. 288-301, 2006.

[20] A. Chakrabarti, T. Zickler, and W.T. Freeman, "Analyzing spatially-varying blur," IEEE Int. Conf. on Comput. Vis. Pattern Recognit., pp. 2512-2519, 2010.

[21] M. Banham and A. Katsaggelos, "Digital image restoration,” IEEE Signal Processing Magazine, pp. 24-41, 1997.

[22] R. Lagendijk, A. Katsaggelos and J. Biemond "Iterative identification and restoration of image," International Conference on Acoustics, Speech, and Signal Processing, pp. 992-995, 1998.

[23] D. Kundur and D. Hatzinakos, "Blind image deconvolution," IEEE Signal Processing Magazine, vol. 13, no. 3, pp. 43-64, 1996.

[24] G. Pavlovic and M. Tekalp, "Maximum likelihood parametric blur identification based on a continuous spatial domain model," IEEE Trans. on Image Processing, vol. 1, no. 4, pp. 496-504, 1992.

[25] J. Jia, "Single image motion deblurring using transparency," IEEE Int. Conf. on Comput. Vis. and Pattern Recognition, pp. 1-8, 2007.

[26] L. Bar, B.Berkels, M.Rumpf and G. Sapiro, "A variational framework for simaltaneous motion estimation and restoration of motion-blurred video," IEEE Int. Conf. on Comput. Vis., pp. 1-8, 2007.

[27] B. Su., S. Lu., and C.L. Tan, "Blurred image region detection and classification," ACM Int. Conf. Multimedia, pp. 1397-1400, 2011.

[28] Elena Lazkano and Basilio Sierra, Progress in Artificial intelligence, Springer Berlin Heidelberg, vol. 2902, pp. 171-183, 2003.

[29] Blur Detection Dataset (2015) Available online at: http://www.cse.cuhk.edu.hk/ leojia/projects/dblurdetect/d ataset.html.

[30] X. Lu, X.Li. and L.Mou, "Semi-Supervised multitask learning for scene recognition," IEEE Trans. Cybernetics, vol. 45, no. 9, pp. 1967-1976, 2015.

[31] Y. Pang., K. Wang, Y. Yuan and K. Zhang, "Distributed object detection with linear SVM," IEEE Trans. Cybernetics, vol. 44, no. 11, pp. 2122-2133, 2014.

[32] Regis Behmo, Arnak Dalalyan, Paul Marcombes and Veronique Prinet, " Towards optimal naïve bayes nearest neighbor," Springer Berlin Heidelberg, pp. 171-184, 2010.

[33] Sancho McCann and David G.Lowe, "Local naive bayes 
nearest neighbor for image classification,'IEEE Conference on Comp. Vision and Pattern Recognition, pp. 3650-3656, 2012.

\section{Authors' Profiles}

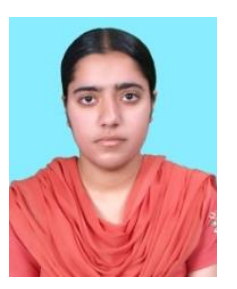

Harjot Kaur: Post-graduate student for M. Tech. degree in Computer Science and Engineering in Sri Guru Granth Sahib World University, Fatehgarh Sahib, Punjab, India.

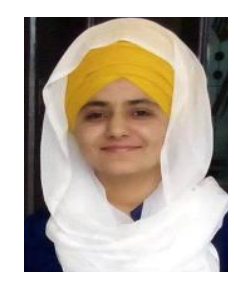

Mandeep Kaur: currently working as Assistant Professor in Computer Science and Engineering Department, Sri Guru Granth Sahib World University, Fatehgarh Sahib, Punjab, India.

How to cite this paper: Harjot Kaur, Mandeep Kaur, "A Hybrid Approach for Blur Detection Using Naïve Bayes Nearest Neighbor Classifier", International Journal of Information Technology and Computer Science(IJITCS), Vol.8, No.12, pp.75-82, 2016. DOI: 10.5815 /ijitcs.2016.12.09 